\title{
NAUSEA AND VOMITING OF PREGNANCY AND HG
}

natuire DISEASE

REVIEWS PRIMERS

Nausea and vomiting of pregnancy (NVP) is common and usually occurs in weeks 6-8 to weeks 16-20 of pregnancy. Hyperemesis gravidarum (HG) can be described as the most severe form of NVP. HG can last the duration of the pregnancy and have complications such as dehydration and nutritional deficiencies.

\section{DIAGNOSIS}

Despite a lack of a consensus definition to distinguish $\mathrm{HG}$ from NVP, and variation in definitions or criteria for $\mathrm{HG}$, current clinical practice is that $\mathrm{HG}$ can be diagnosed in a pregnant woman with severe vomiting and/or severe nausea after other causes have been ruled out. Two tools - the PUQE and the HELP scores - can rate the symptoms of NVP and severe NVP (including $H G)$, respectively, aiding management.

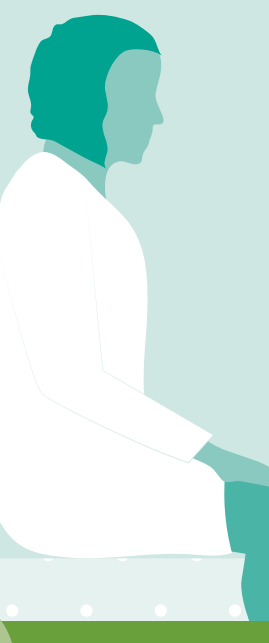

SCREENING

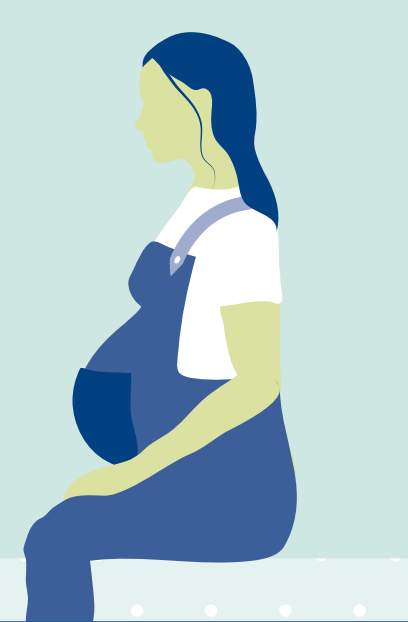

N in primary (that is, general practice) antenatal care is not routine, which can result in delayed treatment. As some of the effects of HG can be serious, patients with worsening NVP and those with HG should be monitored.

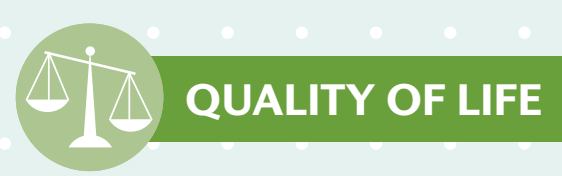

NVP and HG are associated with extreme reduction in quality of life. In particular, HG can be associated with quality of tife. In particular, HG can be associated with substantial maternal and fetal risks and outcomes.

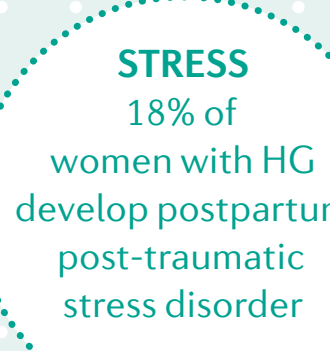

DEHYDRATION AND ELECTROLYT IMBALANCE

$$
\text { Hypokalaemia and }
$$
yponatraemia can lead to cardiac arrhythmias, ventricular fibrillation and cardiac arrest

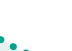
(n)

Variations in definition make quantification of the burden of NVP and HG difficult, but some estimates suggest $~ 70 \%$ of pregnant women experience NVP and a prevalence of $\mathrm{HG}$ of $1.1 \%$ globally. Women $<20$ years of age and those who are pregnant for the first time experience

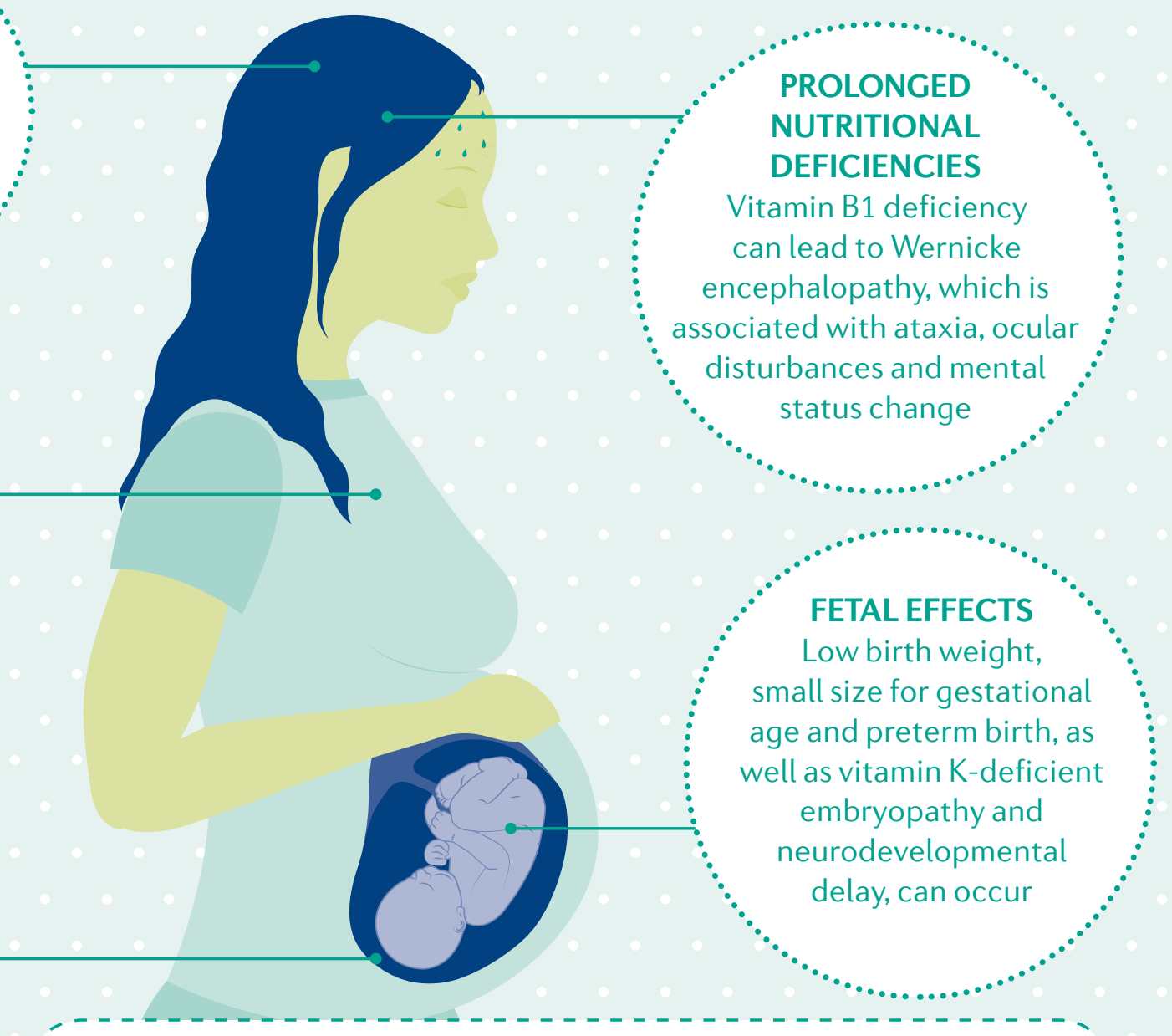

R Dietary and lifestyle changes are the usual first-line treatments, but if symptoms persist, complementary treatments

\section{(such as acupressure) can be introduced. If symptoms worsen,}

pharmacological intervention, fluid and/or nutritional therapy, and/or hospitalization may be required.

\section{NVP at higher rates than others, \\ United Kingdom, annual costs to} and some ethnic differences in NVP have been documented the National Health Service for $\mathrm{NVP}$ and $\mathrm{HG}$ are estimated to be but data are conflicting. In the as high as $€ 62$ million.

\section{NVP is misleadingly referred to as 'morning sickness';} oniy $1.8 \%$ of pregnant women report morning-only symptoms, but $80 \%$ report all-day nausea
For primer vist do 101038/s41572-019-0110-3

\section{MECHANISMS}

The pathogenesis of NVP seems to be multifactorial, involving genetic, endocrine and gastrointestinal factors. Of these factors, the most evidence has been acquired on the hormone GDF15, which was identified in a large genetic study as being associated with NVP and HC. The working hypothesis is that factors including genetic variants, long-term nutrient deprivation, and hyperthyroidism contribute to altered GDF15 levels, resulting in abnormally high levels of GDF15 in the maternal bloodstream. When GDF15 travels to the brain, it signals appetite loss and taste aversion as well as nausea and vomiting, although this has not been definitively proven. Other evidence suggest roles for the GDF15 receptor (GFRAL), thyroid-stimulating hormone, serotonin receptors, the progesteron receptor and insulin-like growth factor binding protein 7 in the aetiology.

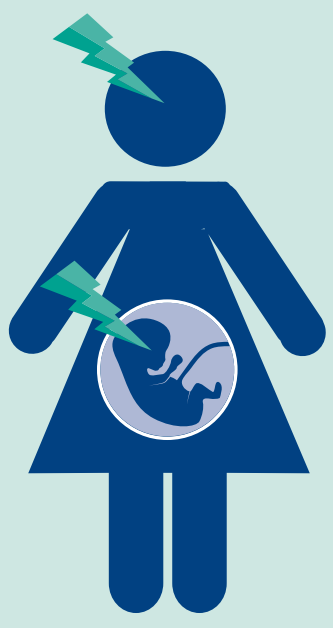

\section{OUTLOOK}

Despite clear effects on quality of life, women with NVP and HG report experiencing a lack of empathy and care, feelings of isolation and lack of understanding and support from healthcare providers. Patients and patient-led organizations are helping shift this narrative and guide research priorities, as well as helping with recruitment to clinical trials and raising funds for research. A growing interest in NVP and HG from a biological perspective will and HG from a biological perspective will
lead to better understanding of the aetiology, identification of risk factors and development of treatments. 